\title{
Indicadores da Implementação do Nível E do MR-MPS em uma Instituição de Pesquisa
}

\author{
Gleison Santos, Mariano Montoni, Reinaldo C. Silva Filho, \\ Anne Elise Katsurayama, David Zanetti, Andrea O. S. Barreto, Ana Regina Rocha \\ COPPE/UFRJ - Universidade Federal do Rio de Janeiro \\ Programa de Engenharia de Sistemas e Computação \\ Av. Horácio Macedo, 2030, Prédio do Centro de Tecnologia, Bloco H, Sala 319 \\ Caixa Postal 68511 - CEP 21941-914 - Rio de Janeiro - RJ - Brazil \\ \{gleison, mmontoni, cabral, anneelisek, zanetti, \\ ansoares, darocha\} @cos.ufrj.br
}

\begin{abstract}
The Software Quality Area of COPPE's Software Engineering Laboratory was evaluated on the level E of MR-MPS in May 2008. It was the first organizational unit appraised on the MR-MPS Level E version 1.2. This paper presents details of this software process improvement initiative implementation including the description of case tools used and the analysis of the experience based on the results presented in measurement reports.

Resumo. Em maio de 2008, a Área de Qualidade de Software do Laboratório de Engenharia de Software da COPPE/UFRJ foi a primeira unidade organizacional a ser avaliada no Nivel E do MR-MPS versão 1.2. Este artigo apresenta os principais detalhes da implementação dessa iniciativa de melhoria de processos, incluindo as ferramentas utilizadas e uma análise da experiência baseada em resultados apresentados nos relatórios de medição.
\end{abstract}

\section{Introdução}

A COPPE/UFRJ é formada por um conjunto de programas de pós-graduação, entre eles o Programa de Engenharia de Sistemas e Computação (PESC). Cada programa é formado por áreas de pesquisa e laboratórios. A área de Engenharia de Software é responsável por atividades de ensino, pesquisa, consultoria e também pelo Laboratório de Engenharia de Software (LENS). O objetivo deste artigo é apresentar os principais detalhes da implementação da iniciativa de melhoria de processos na Área de Qualidade de Software do LENS por meio dos indicadores de medição utilizados. Esta iniciativa foi iniciada em outubro de 2007 e culminou com a avaliação da unidade organizacional no Nível E do MR-MPS versão 1.2 em maio de 2008. Serão discutidas a utilização das ferramentas utilizadas e uma análise crítica da experiência baseada em análises dos indicadores presentes nos relatórios de medição desenvolvidos ao longo de 2008. A próxima seção descreve alguns dos desafios da implementação do Nível E do MR-MPS. Na seção 3 serão relatados detalhes da implementação realizada para cada um dos processos avaliados. Por fim, a seção 4 contém as considerações finais deste trabalho.

\section{Desafios da Implementação do Nível E do MR-MPS}

Apesar de serem importantes mecanismos para fomentar definição, institucionalização e melhoria de processos de software em uma organização, os modelos de maturidade e 
normas internacionais não descrevem de fato os processos a serem seguidos pelas organizações [Emmerich et al. 1999]. A definição destes processos deve ser feita respeitando as características e restrições da organização e depende de muitos fatores, incluindo o domínio da aplicação, a estrutura e o tamanho da organização [Chrissis et al. 2006]. O MPS.BR [SOFTEX 2007b] é o programa para Melhoria de Processo do Software Brasileiro e define um modelo de referência para melhoria e avaliação de processos de software de forma a atender as necessidades de negócio de empresas brasileiras. O Nível E do MR-MPS (Modelo de Referência do MPS.BR) tem como foco principal a padronização dos processos da organização, por meio da definição de processos padrão [SOFTEX 2007a]. Estes devem ser definidos a partir dos processos e melhores práticas já existentes na organização, o que constitui o primeiro passo de uma contínua avaliação e melhoria dos processos. A definição de processos padrão inclui, além dos processos do nível $\mathrm{E}$, todos os processos que pertencem aos níveis $\mathrm{G}$ e $\mathrm{F}$ do MR-MPS. Como exigência para a evolução ao nível E do MR-MPS tem-se ainda a construção da biblioteca de ativos de processo e do repositório de medidas. Neste nível, para garantir a institucionalização dos processos e a correção no seu uso, deve-se institucionalizar a gerência de recursos humanos por meio da identificação, desenvolvimento e/ou contratação de indivíduos que possuam os conhecimento e habilidades necessários para atender os objetivos estratégicos da organização. A organização deve, também, implementar uma estratégia de gerência de ativos reutizáveis por meio da reutilização de produtos de trabalho projetados para utilização em múltiplos contextos.

\section{A Implementação do Nível E do MR-MPS na Área de Qualidade de Software do Laboratório de Engenharia de Software da COPPE/UFRJ}

No final de 2007, os membros da II e da IA COPPE/UFRJ decidiram pela implementação do nível E do MR-MPS versão 1.2 na Área de Qualidade do LENS da COPPE/UFRJ. Essa implementação visa à construção de um ambiente de alta maturidade que possa ser utilizado como laboratório para a aplicação de estratégias de implementação de melhoria de processos de software. Este ambiente possibilitará a aplicação de técnicas de melhoria de processos de software e a aquisição de conhecimentos ainda não tão comuns no mercado, onde a maioria das organizações ainda se encontra nos níveis mais baixos de maturidade. $\mathrm{O}$ conhecimento advindo desta aplicação será utilizado em organizações cliente com o intuito de aumentar a probabilidade de sucesso das iniciativas de melhoria. Lições aprendidas nesta iniciativa, incluindo pontos fortes e dificuldades enfrentadas, são descritas em [Santos et al. 2009].

A Área de Qualidade de Software do LENS possui um portfólio de projetos que engloba customizações do ambiente de gestão do conhecimento CORE-KM [Galotta et al. 2004] para clientes externos; evolução do ambiente de desenvolvimento de software TABA [Montoni et al. 2006b], utilizado por organizações para apoiar iniciativas de melhoria; e desenvolvimento dos produtos de software que fazem parte de dissertações de mestrado e teses de doutorado. Estes tipos de produtos fizeram parte do escopo da avaliação no nível E do MR-MPS. Muitas das práticas previstas pelo modelo já eram utilizadas pela equipe. Além disso, a estratégia de implementação da COPPE/UFRJ, a SPI-KM [Santos et al. 2007], é baseada no uso de ferramentas, em especial a Estação TABA [Montoni et al. 2007], e já foi utilizada em vários casos de sucesso, incluindo organizações avaliadas no nível E do MR-MPS (por exemplo, [Nunes et al. 2006; Scheid et al. 2007]) e nível 3 do CMMI (por exemplo, [Ferreira et al. 2007]). 


\subsection{Gerência de Projetos e Gerência de Requisitos}

As atividades relacionadas à Gerência de Projetos foram realizadas com o auxílio das ferramentas de planejamento, monitoração e controle existentes na Estação Taba, para, por exemplo, planejamento de cronograma, custos, recursos humanos e riscos [Montoni et al. 2007]. Para as atividades relacionadas à Gerência de Requisitos foi utilizada a ferramenta Enterprise Architect. É importante notar que, apesar de não obrigatórias no Nível E do MR-MPS, inspeções foram realizadas em documentos chave (como planos do projeto, especificação de requisitos e planos e relatórios de testes) de forma a aumentar a eficácia dos processos e visando à redução do retrabalho e quantidade de defeitos nos artefatos gerados. Durante todo o ano de 2008, a monitoração do processo de Gerência de Requisitos evidenciou que os requisitos mantiveram-se estáveis durante todo o período (reflexo dos esforços para um correto e eficiente levantamento e verificação de requisitos). $\mathrm{O}$ indicador relacionado à densidade de defeitos (número de defeitos / tamanho do software em Pontos por Casos de Uso) nos testes conseguiu mostrar a eficácia dos procedimentos adotados, como pode ser visto nos dados apresentados na Figura 1 que mostra grande número de defeitos encontrados nos testes de software resolvidos antes da homologação externa. Foram reportados erros na homologação externa apenas no projeto SS_2008_011 (mas com uma densidade próxima do zero), todos os erros encontrados no teste de software dos demais projetos foram todos retirados antes da homologação (inclusive do projeto SS_2008_032 cuja densidade de erros era próxima de 1). Métricas para quantificar defeitos foram escolhidas para começar a entender o comportamento dos processos de engenharia, já visando a uma avaliação do nível C do MR-MPS e, também, para criar uma base histórica visando à futura análise quantitativa, necessária para uma avaliação do nível A do MR-MPS.BR.
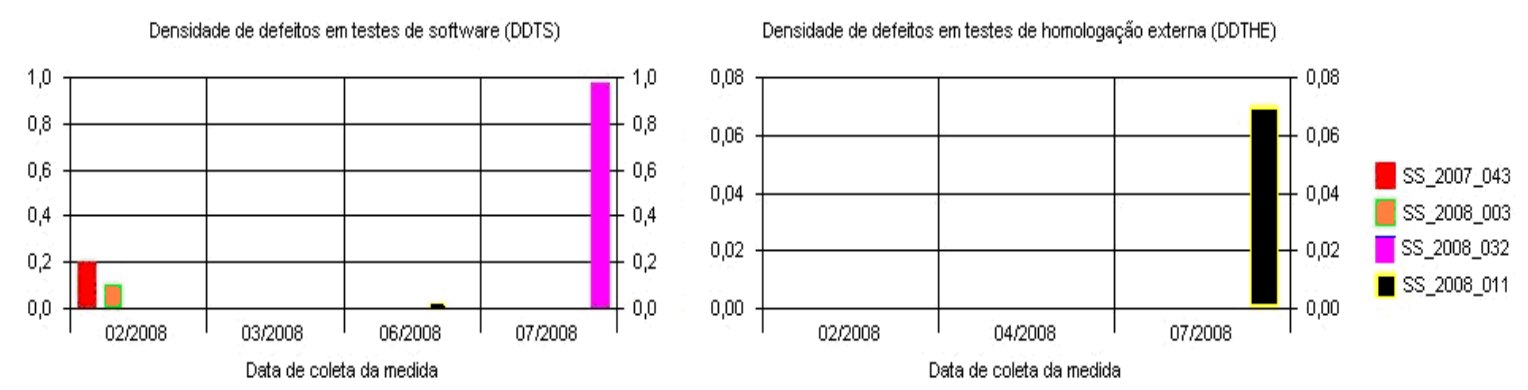

Figura 1. Análise das métricas de defeito

A análise da precisão de estimativas de cronograma e esforço dos projetos mostrou problemas no processo de estimativas do prazo e esforço total do projeto, como visto na Figura 2. Os problemas de prazo foram decorrentes, em sua maioria, da superalocação dos membros da equipe em várias atividades, com o intuito de cumprir o cronograma da avaliação, e ao prazo curto dos projetos mostrados que transformam poucos dias de desvio em um grande valor porcentual. A precisão da estimativa de esforço foi muito ruim durante o início do ano devido à falta de uma base histórica adequada, que só foi construída após os projetos terem sido finalizados. Além disso, alguns problemas pontuais relacionados a mudanças de requisitos e falta de conhecimento da equipe na tecnologia utilizada em um dos projetos também causou desvios significativos. Devido a estes problemas, a análise das medidas passou a ser feita em uma granularidade mais fina (por exemplo, fase ou atividade do processo) para aumentar a visibilidade do ponto onde o problema se mostrava presente pela primeira vez. 

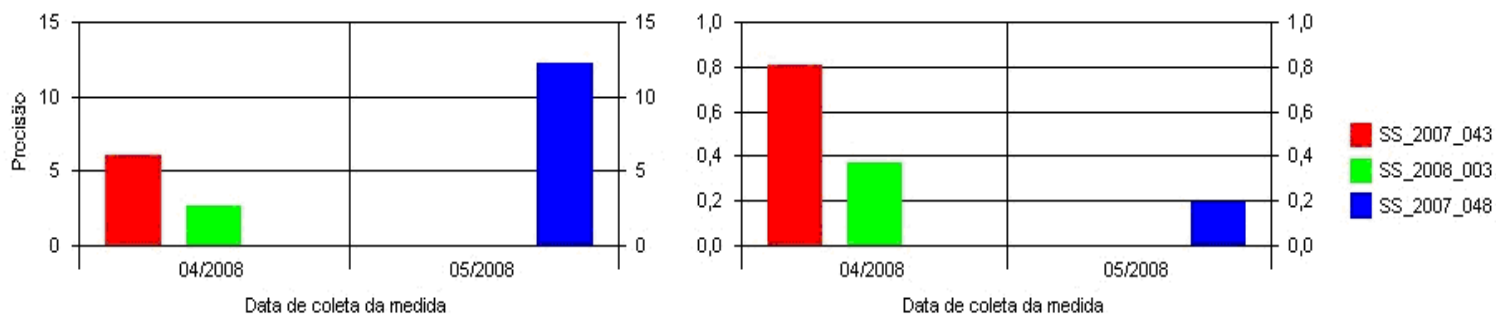

Figura 2. Análise das métricas de precisão de estimativas

\subsection{Gerência de Configuração, Garantia da Qualidade e Medição}

Atividades de Gerência de Configuração, tanto dos projetos de desenvolvimento quanto dos processos de execução organizacional, foram realizadas com o auxílio da ferramenta GConf [Santos et al. 2008] da Estação Taba. A GConf é responsável pela gerência dos artefatos com nível de controle alto, ou seja, que só podem ser alterados após a execução de um processo formal de alteração, como, por exemplo, requisitos acordados com os clientes. Além disso, documentos com nível de controle de baixo (ou seja, podem ser alterados livremente) tiveram versões controladas com o uso do SubVersion. Auditorias de Gerência de Configuração são executadas tanto no contexto dos projetos quanto no organizacional pelo Grupo de Gerência de Configuração (composto por 1 pessoa). As atividades de Garantira da Qualidade foram realizadas pelo Grupo de Garantia da Qualidade do Processo e do Produto (GQPP, composto por 2 pessoas). As avaliações de produto foram realizadas com o uso de checklists específicos que buscam analisar a forma e o conteúdo dos documentos. As avaliações de processo eram realizadas nos projetos em conjunto com cada avaliação de produto com auxílio das ferramentas AdaptPro e Acknowledge [Montoni et al. 2006a]. Para os processos executados fora do contexto dos projetos, as avaliações de processo são feitas mensalmente. As responsabilidades do GQPP também incluem a elaboração de um Relatório de Situação dos Processos mensalmente que contém informações sobre a aderência, pertinência e possíveis melhorias de todos os processos analisados. As atividades de Medição e Análise são realizadas pelo Grupo de Medição (composto por 1 pessoa) mensalmente. O Relatório de Medição e Análise é apresentado em conjunto com o Relatório de Situação dos Processos à Alta Gerência, e participação de todos os grupos organizacionais envolvidos, visando a uma apreciação conjunto de várias fontes para melhoria dos processos. As medidas são coletadas, armazenadas e analisadas com o auxílio das ferramentas MedPlan e Metrics [Schnaider et al. 2004]. Os gráficos de barra apresentados neste artigo são produzidos pela ferramenta Metrics. A base histórica de estimativas da organização foi construída com base nos registros da Metrics. A partir das informações consolidadas, foi gerada uma planilha de estimativas no Excel para ser utilizada no planejamento dos projetos.

As métricas selecionadas para monitoração dos processos de Garantia da Qualidade e Gerência de Configuração procuraram observar o número de não conformidades encontradas nas avaliações de qualidade e nas auditorias de gerência de configuração além da taxa de resolução destes problemas. Durante todo o período foi possível observar que as avaliações de qualidade de produto aconteceram conforme planejado e os valores obtidos pelas medições estavam dentro da meta estabelecida (a isso também 
pode ser creditado, de alguma forma, o bom desempenho das medidas de defeito mencionadas anteriormente). As métricas relacionadas a avaliações de aderência ao processo e auditorias de gerência de configuração sofreram desvios decorrentes dos desvios das estimativas dos projetos, mas a qualidade dos produtos não foi afetada por isso. As métricas relacionadas ao processo de Medição procuravam observar quantas das medidas estavam dentro da meta estabelecida. No geral, as medidas conseguiram atender as metas, porém foi comum durante o ano a falta de dados para a análise das medidas. Isso se deveu, por exemplo, a não existência de projetos executando atividades que possibilitassem a coleta dos dados previstos para todas as medidas ou à estabilidade dos processos depois de algum tempo no caso das medidas relacionadas à melhoria de processos. Assim, estas medidas, apesar do que pode parecer à primeira vista, foram consideradas adequadas à monitoração dos processos e permitiram a identificação ou confirmação de problemas na execução dos processos nos meses seguintes.

\subsection{Definição, Avaliação e Melhoria do Processo Organizacional}

A Biblioteca de Ativos de Processos da Estação Taba [Santos et al. 2008] foi utilizada como o principal repositório dos processos, da política organizacional e dos demais ativos organizacionais definidos com nível de controle alto de gerência de configuração. Os itens de controle baixo foram também armazenados no SubVersion, que também serviu como mecanismo para divulgação das baselines organizacionais. A definição do processo padrão de desenvolvimento de software foi feita utilizando-se a ferramenta Config [Villela et al. 2004] da Estação Taba e a adaptação destes processos aos projetos de desenvolvimento foi feita pela ferramenta AdaptPro [Villela et al. 2004]. As solicitações de melhoria em todos os ativos, com nível baixo ou alto, foram gerenciadas através da utilização do Bugzilla. Todas as solicitações de melhoria são analisadas por um membro do Grupo de Processos (composto por 2 pessoas) e, se pertinentes, podem ser implementadas imediatamente (por exemplo, mudanças simples em templates) ou adiadas para o próximo ciclo de melhoria (por exemplo, grandes mudanças nos processos). Planos, incluindo um cronograma detalhado comum a todas as atividades organizacionais, foram gerados para gerenciar estas atividades. As medidas relacionadas a estes processos procuram analisar as melhorias no processo organizacional (conforme mostra a Figura 3) e o número de projetos que utilizam os processos padrão da empresa (Obs.: as diferentes cores mostradas nesta figura não têm semântica própria). Foi notado um grande número de melhorias no período inicial da implantação dos processos que diminuiu com o passar do tempo demonstrando a maior estabilidade e maturidade. Também foi notado que as melhorias apresentadas a partir de um determinado momento implicavam em mudanças grandes nos processos e, por isso, foram programadas para serem implementadas em um novo ciclo de melhoria.

Número de Melhorias Propostas (NMP)

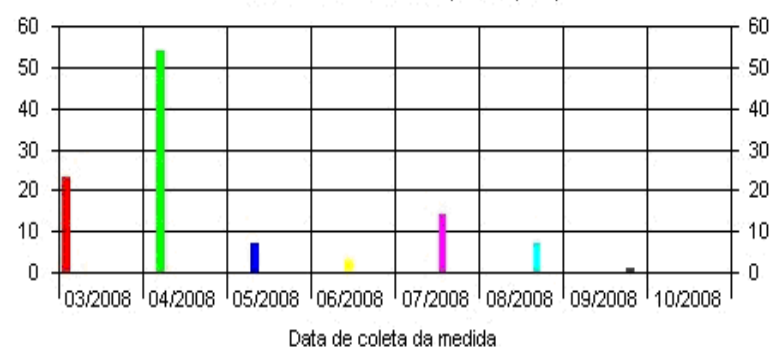

Número de Melhorias Implementadas (NMM)

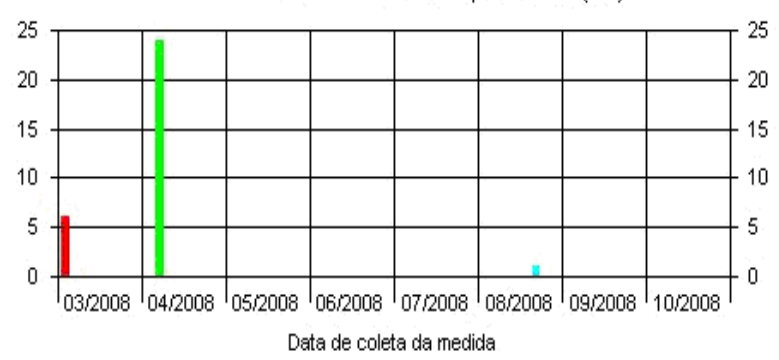

Figura 3. Medidas relacionadas a melhorias dos processos 


\subsection{Gerência de Reutilização}

Para atender aos processos do nível E do MR-MPS, houve a necessidade de definição de uma estratégia para as atividades de gerência de reutilização. A estratégia definida é composta por um processo de Gerência de Reutilização e algumas ferramentas de apoio [Silva Filho et al. 2008]. O processo de Gerência de Reutilização é composto pelas seguintes atividades: Disponibilizar Ativo, Manter Ativos Reutilizáveis e Notificar Interessados. O processo de Gerência de Reutilização atua no âmbito organizacional e ao longo dos projetos. No âmbito organizacional são definidos, avaliados, mantidos e disponibilizados ativos reutilizáveis relativos aos processos organizacionais. Estes ativos reutilizáveis também são definidos da mesma forma ao longo dos projetos de desenvolvimento e manutenção de software. Dentre as principais dificuldades para implementação do processo de GRU, destacaram-se: (i) a definição de uma estratégia não-intrusiva, ou seja, que não tivesse impacto nas atividades cotidianas das pessoas da unidade organizacional e que facilitasse a integração com os outros processos; e (ii) a identificação de métricas úteis à monitoração e controle do processo. As atividades gerenciais foram realizadas pelo Grupo de Reutilização (composto por 1 pessoa). As aceitações dos ativos foram realizadas pelo Grupo de Processo e as certificações foram realizadas pelo GQPP. Para que a monitoração e controle do processo fosse realizada de forma satisfatória, foi necessário identificar métricas que pudessem aferir se o processo estava atingindo seus objetivos e que fossem úteis na identificação de oportunidades de melhoria no processo, conforme pode ser visto na Figura 4. Cientes da importância de equilibrar a representatividade das métricas selecionadas com o custo de medição associado, foram selecionados apenas dois indicadores: um para acompanhar a taxa de reutilização dos ativos e outro para acompanhar a evolução da base de ativos reutilizáveis. Estes indicadores também serviram de referência para outros aspectos de interesse da alta gerência. Percebeu-se, por exemplo, que o total de reutilizações possui relação direta com a carga de trabalho da unidade organizacional, em outras palavras quanto maior o número de projetos e mais rápido evoluem, maior o número de reutilizações.

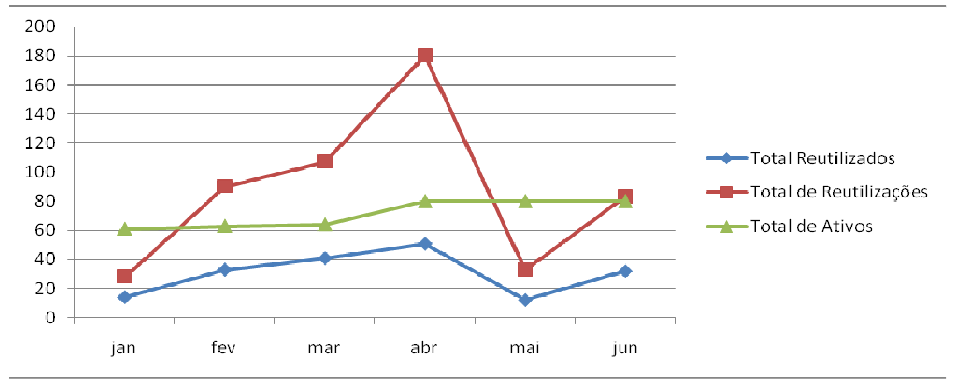

Figura 4. Total de ativos reutilizados e total de reutilizações entre jan-jun/2008

\subsection{Gerência de Recursos Humanos}

As atividades de Gerência de Recursos Humanos foram executadas com base num Plano de Capacitação. Este plano prevê que, para atender aos diversos tipos de tarefas sob sua responsabilidade, a equipe é submetida a um programa de capacitação que vai se aprofundando de acordo com o estágio acadêmico. Busca-se atingir um nível bastante profundo de conhecimento e experiência em Engenharia de Software, com ênfase em qualidade de software, ao mesmo tempo em que todo o conhecimento técnico para realizar as atividades específicas dos projetos de desenvolvimento de software. Como a- 
poio à capacitação foi elaborado um Plano de Treinamento visando ao aprimoramento em atividades específicas do desenvolvimento de software (como, por exemplo, linguagens de programação). Além disso, foi instituída a realização de Workshops de Disseminação do Conhecimento do Processo de Desenvolvimento visando à transmissão de conhecimento entre diferentes pessoas da equipe e à evolução da base de conhecimento armazenada na ferramenta Acknowledge [Montoni et al. 2006a]. Esta ferramenta implementa um processo de aquisição e empacotamento de conhecimento e é fortemente utilizada durante os projetos de desenvolvimento para disponibilização da base de conhecimento da organização composta por itens como diretrizes e lições aprendidas. Além disso, a ferramenta Sapiens [Santos et al. 2004], integrada à ferramenta de planejamento de recursos humanos para os projetos, possibilita a manutenção da base de competências da organização. Grupos de discussões via e-mail foram institucionalizados para a discussão de assuntos pertinentes com especialistas da organização em Processos de Software e Tecnologias .Net e Java. Avaliações de pessoas com base em critérios objetivos, assim como avaliações da efetividade dos treinamentos realizados, são realizadas durante avaliações post mortem dos projetos. Estas avaliações servem, portanto, para realimentar a base de competências, a base de conhecimento e, também, proveem importantes solicitações de melhorias para os processos. A principal medida para monitoração do processo de gerência de conhecimento adotado. Percebeu-se um grande um grande pico na submissão e aprovação de novos itens de conhecimento nos meses de março e maio de 2008 decorrentes da institucionalização dos processos (fato também corroborado pelos indicadores exibidos na Figura 3) e da necessidade de se definir formas de operacionalizar e institucionalizar uma forma única de execução de certas atividades que eram descritas de forma pouco clara ou específica no processo de desenvolvimento de software (por exemplo, definição de diretrizes para explicitar regras de adaptação do processo padrão no que se refere à obrigatoriedade da definição de diagramas de sequencia durante a construção do modelo de análise e projeto).

\section{Considerações Finais}

Este artigo apresentou os principais detalhes da implementação do Nível E do MR-MPS na Área de Qualidade do Laboratório de Engenharia de Software da COPPE/UFRJ. Apesar de concluída satisfatoriamente em todos os requisitos do nível E do MR-MPS, a avaliação permitiu a identificação de oportunidades de melhoria. Essas oportunidades serão consideradas nos próximos ciclos de melhoria a serem implementados. A Área de Qualidade do LENS objetiva os níveis mais altos do MPS.BR com o intuito de criar um ambiente de alta maturidade para estudo das técnicas de melhoria de processos de software. A meta atual é alcançar o nível C do MR-MPS em 2009 e o nível A em 2010.

\section{Referências}

Chrissis, M.B., Konrad, M., Shrum, S. (2006), CMMI (Second Edition): Guidelines for Process Integration and Product Improvement, Addison Wesley Professional.

Emmerich, W., Finkelstein, A., Fuggetta, A., et al. (1999), "Software Process Standards, Assessments and Improvement", pp. 15-26, 1999.

Ferreira, A.I.F., Santos, G., Cerqueira, R., et al. (2007), "Applying ISO 9001:2000, MPS.BR and CMMI to Achieve Software Process Maturity: BL Informatica's Pathway". In: 29th Int. Conference on Software Engineering (ICSE), pp. 642651, Minneapolis, USA, May.

Silva Filho, R.C., Katsurayama, A.E., Santos, G., et al. (2008), "Deploying Software 
Reuse Management at COPPE/UFRJ Software Engineering Laboratory". In: Workshop on Software Reuse Efforts - WSRE 2008, Recife - PE.

Galotta, C., Zanetti, D., Rocha, A.R. (2004), "Organizational Learning Based on a Customizable Environment for Knowledge Management Using Intranet", v. 2, pp. 2626-2633, Washington, EUA.

Montoni, M., Santos, G., Figueiredo, S., et al. (2006a), "Uma Abordagem de Garantia de Qualidade de Processos e Produtos de Software com Apoio de Gerência de Conhecimento na Estação TABA". In: V Simpósio Brasileiro de Qualidade de Software - SBQS 2006, pp. 87-99, Vila Velha - ES.

Montoni, M., Santos, G., Rocha, A.R., et al. (2006b), "Taba workstation: Supporting software process deployment based on CMMI and MR-MPS.BR", v. 4034 NCS, pp. 249-262, Amsterdam, Netherlands.

Montoni, M., Santos, G., Rocha, A.R., et al. (2007), "MPS Model and TABA Workstation: Implementing Software Process Improvement Initiatives in Small Settings". In: Fifth Workshop on Software Quality held in conjunction with the 29th Int. Conference on Software Engineering (ICSE), Minneapolis, USA, May.

Nunes, E.D., Pinto, R., Rocha, A.R., et al. (2006), "MPS.BR Nível E - Uma Avaliação em Verde e Amarelo". In: V Simpósio Brasileiro de Qualidade de Software SBQS 2006, pp. 318 - 325, Vila Velha - ES, Junho 2006.

Santos, G., Katsurayama, A.E., Montoni, M., et al. (2009), "Lições Aprendidas em uma Iniciativa de Melhoria de Processos de Software sob Diferentes Perspectivas: Membros da Organização, Implementadores e Avaliadores". In: VIII Simpósio Brasileiro de Qualidade de Software - SBQS 2009, Ouro Preto - MG, Junho 2009.

Santos, G., Montoni, M., Figueiredo, S., et al. (2008), "Uma Estratégia de Gerência de Configuração de Ativos de Processos de Software Apoiada por um Ambiente de Engenharia de Software Centrado em Processos". In: VII Simpósio Brasileiro de Qualidade de Software - SBQS 2008, Florianópolis - SC, Junho 2008.

Santos, G., Montoni, M., Figueiredo, S., et al. (2007), "SPI-KM - Lessons Learned from Applying a Software Process Improvement Strategy Supported by Knowledge Management", Product-Focused Software Process Improvement.

Santos, G., Villela, K., Schnaider, L., et al. (2004), "Building Ontology Based Tools for a Software Development Environment". In: Advances in Learning Software Organizations, pp. 19-30, Banff, Canada.

Scheid, M., Pessoa, M.V., Gomes, R.F., et al. (2007), "Implantação do MR-MPS Nível E no Centro de Computação da Aeronáutica de São José dos Campos", ProQuality (UFLA).

Schnaider, L., Santos, G., Montoni, M., et al. (2004), "Uma Abordagem para Medição e Análise em Projetos de Desenvolvimento de Software". In: III Simpósio Brasileiro de Qualidade de Software - SBQS 2004, Brasília - DF.

SOFTEX, "MPS.BR - Guia de Implementação - Parte 3: Nível E". In: www.softex.br/mpsbr, acessado em.25/01/2009

SOFTEX, "MPS.BR - Guia Geral". In: www.softex.br, acessado em.25/01/2009

Villela, K., Santos, G., Montoni, M., et al. (2004), "Definição de Processos em Ambientes de Desenvolvimento de Software Orientados a Organização". In: III Simpósio Brasileiro de Qualidade de Software - SBQS 2004, pp. 4-18, Brasília DF. 